\title{
Robust posture tracking control of stable coaxial two-wheeled AGV using the approximate inverse system and LMI
}

\author{
Minoru Sasaki $^{a^{*}}$, Hideaki Tochigi ${ }^{a}$, Waweru Njeri ${ }^{\mathrm{b}}$, Hiroyuki Hayashi ${ }^{\mathrm{a}}$, \\ Joseph Muguro $^{b}$, Kojiro Matsushita ${ }^{a}$ and Harrison Ngetha ${ }^{b}$ \\ ${ }^{a}$ Department of Mechanical Engineering, Faculty of Engineering, \\ Gifu University, 1-1 Yanagido, Gifu, 501-1193, Japan \\ ${ }^{\mathrm{b}}$ Department of Electrical \& Electronic Engineering, School of Engineering, \\ Dedan Kimathi University of Technology, P.O Box 657-10100, Nyeri, Kenya \\ sasaki@gifu-u.ac.jp
}

\begin{abstract}
This paper describes the design of the posture controller for two-wheeled AGV. The objective is to improve the posture stability when running on ramps by balancing between vehicle body attitude angle and wheel angular velocity target value. The effectiveness of the controller by simulation and experiment was confirmed.
\end{abstract}

Key Words: coaxial two-wheeled AGV, LMI, inverse system, robust control. 


\section{Introduction}

Recently, the development of coaxial two-wheeled robot has been on the rise in the field of personal mobility and mobile robots. Robot movement mechanism can be multi-legged types like a human foot or a four-wheel type like a car. Depending on the movement model, speed and required rotation space are affected. The coaxial two-wheeled vehicle, which is a two-wheeled moving mechanism that enables compact turning on the spot-rotation around the centre of the vehicle body (super turning), is very suitable for use in limited spaces such as indoors and homes. Although the moving mechanism has various advantages, many coaxial two-wheeled vehicles have a centre of gravity above the top like an inverted pendulum. Therefore, a posture controller is indispensable because the posture is not stable. In other words, because the system is an unstable system without

the controller, if an abnormality occurs in any one of the control systems such as the power supply, sensor, or actuator, there is a risk of falling.

Another challenge facing mobility robots is about safety. Recently, in robot development, reliability design based on intrinsic safety design has attracted attention, and the above-mentioned problem is also required in the operation in the symbiotic space between robots and humans.

To deal with such problems, (Jian-Wei \& Xiao-Gang, 2009; Deng, Inoue, Sekiguchi, \& Jiang, 2010) and other researchers have proposed a design that places the overall centre of gravity of the vehicle below the centre of rotation of the wheel. The proposal resulted in a stable coaxial two-wheeled vehicle that requires restoring force and guarantees stability during static balancing. Although the attitude controller was added to this physical restoring force, running performance was not optimal. The major challenges with conventional stable coaxial two-wheeled vehicles include dynamics such as running on rough terrain, acceleration / 
deceleration, and stopping. As a result, there were stability problems in and deviations during static balancing on rough terrain.

In response to these problems, (Chan et al., 2013),(Dai et al., 2015) developed a mechanism that can solve the above-mentioned problems by dividing the vehicle body vertically, adding one degree of freedom of control at a position higher than the axle, and controlling the centre of gravity. The scheme succeeded in eliminating deviation from the conventional model by adopting a mechanism that adds a joint in the car-body integration and divided the body vertically such that each of this link can be controlled individually. The approach trades-off stability on rough terrains such as ramps with attitude angle stability and target speed. The reason is that a controller with only a feedback system can guarantee the stability of an unstable system, suppress the influence of disturbance, and eliminate modelling errors. However, target value robustness is difficult with only the feedback term. It is considered that target value robustness and stability guarantee cannot be achieved at the same time with the current state feedback control system alone.

As various facilities become barrier-free, there will still be many indoor environment facilities with slopes and stairs. Even in such facilities, mobility robots equipped with coaxial two wheels will be ideal based on the advantages highlighted herein. Similarly, movement and control following the target speed are naturally required. Therefore, in this study, robust control that guarantees robustness against disturbance is designed as a feedback controller. The feedforward term by the inverse system is used for input shaping to eliminate the trade-off is used as a pre-compensator. The added controller was designed, and a controller was designed to solve the conventional problems. In this research, the attitude controller design of the stable coaxial two-wheeled robot to be controlled is designed by model-based development. The state-space model of the controlled object is derived, and the attitude controller using the model is designed using MATLAB / Simulink. After that, a running 
simulation was performed to show the effectiveness of the designed controller, and after the validity of the controller was confirmed, an implementation experiment was conducted in an actual system to evaluate and examine the system.

\section{Modeling}

In this study, a state space model that considers the road slope angle as a disturbance term similar to term used in a previous study (Chan et al., 2013) as a state variable was used for control design.

$$
\left\{\begin{array}{c}
\dot{x}(t)=A x(t)+B u(t)+B_{\omega} \omega(t) \\
y(t)=C x(t)
\end{array}\right.
$$

Where $A=\left[\begin{array}{cc}0_{3 \times 3} & I_{3 \times 3} \\ -\alpha^{-1} \gamma & -\alpha^{-1} \beta\end{array}\right], B=\left[\begin{array}{c}0_{3 \times 2} \\ \alpha^{-1} \delta\end{array}\right], C=\left[I_{6 \times 6}\right], B_{\omega}=\left[\begin{array}{c}0_{3 \times 1} \\ -\alpha^{-1} \epsilon\end{array}\right]$

$$
\alpha=\left[\begin{array}{lll}
\alpha_{11} & \alpha_{12} & \alpha_{13} \\
\alpha_{21} & \alpha_{22} & \alpha_{23} \\
\alpha_{31} & \alpha_{32} & \alpha_{33}
\end{array}\right] \beta=\left[\begin{array}{ccc}
C_{p 1} & 0 & 0 \\
-C_{p 2} & C_{p 2} & 0 \\
0 & 0 & C_{c}
\end{array}\right]
$$

$$
\gamma=\left[\begin{array}{ccc}
M_{p 1} g l_{1} & -M_{p 2} g l_{2} & 0 \\
0 & -M_{p 2} g l_{2} & 0 \\
0 & 0 & 0
\end{array}\right], \delta=\left[\begin{array}{cc}
0 & 0 \\
a_{p 2} & 0 \\
0 & a_{c}
\end{array}\right]
$$

$$
\varepsilon=\left[\begin{array}{c}
\left(M_{p 1}+M_{p 2}+M_{c}\right) g r \\
0 \\
0
\end{array}\right]
$$

$$
\begin{aligned}
& \alpha_{11}=\left(M_{p 1}+M_{p 2}+M_{c}\right) r^{2}+M_{p 1} l_{1}^{2}-2 M_{p 1} r l_{1}+M_{p 2} r l_{2}+J_{p 1}+J_{c} \\
& \alpha_{12}=\left(M_{p 2} l_{2}^{2}+M_{p 2} r l_{2}+J_{p 2}\right) \\
& \alpha_{13}=\left(M_{p 1}+M_{p 2}+M_{c}\right) r^{2}-M_{p 1} r l_{1}+M_{p 2} r l_{2}+J_{c} \\
& \alpha_{21}=M_{p 2} r l_{2} \\
& \alpha_{22}=M_{p 2} l_{2}^{2}+J_{p 2} \\
& \alpha_{23}=M_{p 2} r l_{2} \\
& \alpha_{31}=\left(M_{p 1}+M_{p 2}+M_{c}\right) r^{2}-M_{p 1} r l_{1}+J_{c}
\end{aligned}
$$


$\alpha_{32}=M_{p 2} r l_{2}$

$\alpha_{33}=\left(M_{p 1}+M_{p 2}+M_{c}\right) r^{2}+J_{c}$

In addition, in case of a model in which the vehicle body that is integrated, the posture angle of the vehicle body that is in an equilibrium state is uniquely determined. In the case of the model in which the vehicle body is divided vertically, it is in an equilibrium state. There are concerns about the occurrence of steady-state deviations because the body posture angle is infinite. Therefore, in this study, as in the previous study, the controller is designed using an expansion system that is servo-extended by adding an integrator to the attitude angle $\left(\theta_{1}+\theta_{2}\right)$ and wheel rotation angular velocity $\dot{\phi}$. The reference variables for $\left(\theta_{1}+\theta_{2}\right)$ and $\dot{\phi}$ are referred to as $\left(\theta_{1}+\theta_{2}\right)_{\text {ref }}$ and $\dot{\phi}_{\text {ref }}$ respectively.

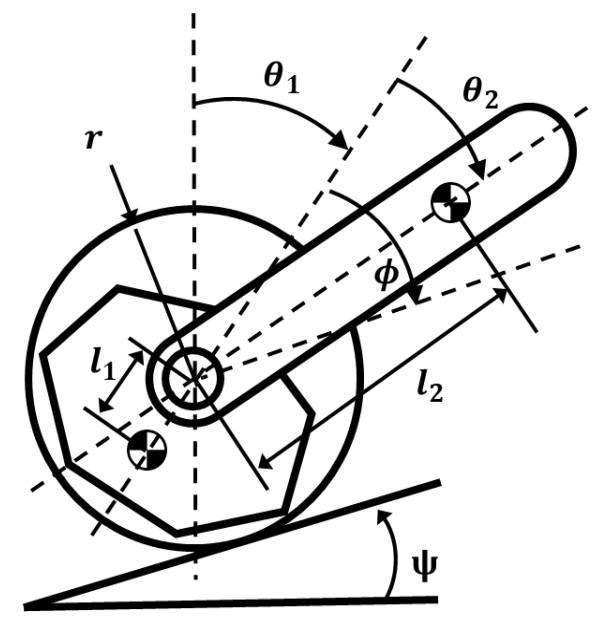

Figure. 1 Coaxial two-wheeled vehicle model.

\section{Controller design}

\subsection{Robust control}

The dynamic characteristics of the controlled object cannot be expressed completely, and a modelling error is likely to occur. Therefore, there is no strict guarantee that the control system designed for a real system will work well, and in the worst case, the closed-loop system may become unstable. Therefore, it is necessary to design a controller that achieves the desired control performance for all models belonging to the set. Robustness in control is 
to guarantee the control effect including the stability and response characteristics of the controlled object even when the control elements such as the controller and the plant are perturbed. Robust control refers to the fluctuation of the perturbed plant. In this research, a feedback system controller that satisfies $H_{2}$ performance is designed by linear matrix inequality (LMI) to realize $H_{2}$ control performance in compensating robustness against impulsive disturbance with state feedback(Liu \& Dong, 2018).

\section{Linear matrix inequality (LMI)}

This study is a multi-purpose control with multiple control specifications to guarantee the stability of the system and to compensate the robustness against the road surface inclination and angle disturbance. At the same time, the paper seeks to present a general-purpose controller that can be added to the controller derivation when a new control requirement appears. Therefore, reduced form LMI that can satisfy multiple control requirements that satisfy $H_{2}$ performance and guarantee stability against tilt angle disturbance was used. A multipurpose and versatile controller is realized by reducing all the control specifications of this research to LMI conditions as shown below.

$$
\begin{gathered}
\min _{\text {s.t }}^{\gamma_{2}}\left[\begin{array}{cc}
\mathrm{He}\{\mathrm{AX}+\mathrm{BY}\} & (\mathrm{CX}+\mathrm{DY})^{\mathrm{T}} \\
(\mathrm{CX}+\mathrm{DY}) & -\mathrm{I}
\end{array}\right] 0, \\
{\left[\begin{array}{cc}
\mathrm{Z} & \mathrm{B}_{\omega}^{\mathrm{T}} \\
\mathrm{B}_{\omega} & \mathrm{X}
\end{array}\right]>0, \mathrm{X}>0, \operatorname{trace}(\mathrm{Z})<\gamma_{2}{ }^{2} .}
\end{gathered}
$$

\subsection{Binary search}

In order to derive a decision variable from LMI conditions and design a state feedback controller, a search algorithm is needed to derive $\gamma_{2}$, which is the objective function to be minimized. In this study, binary search was used as a search algorithm. First, the upper and lower limits are set so that the optimal solution for $\gamma_{2}$ is included in the derivation flow. Then, the binary value is derived for the set upper and lower limits, and the LMI is solved for the binary value. If the LMI for the derived binary value $\gamma_{2}$ has a solution, the search 
algorithm is looped over the range of $\gamma_{2}$ with the binary value as the upper limit because there may be $\gamma_{2}$ satisfying the LMI condition with a value smaller than the binary value. On the other hand, if the LMI condition for the derived binary value $\gamma_{2}$ does not have a solution, it is determined that there is no $\gamma_{2}$ satisfying the LMI condition below the binary value, and the binary search is performed with the binary value as the lower limit. This study involved searching for an optimal solution using an algorithm that minimizes $\gamma_{2}$ when the difference between the upper and lower limits is less than $10^{-2}$.

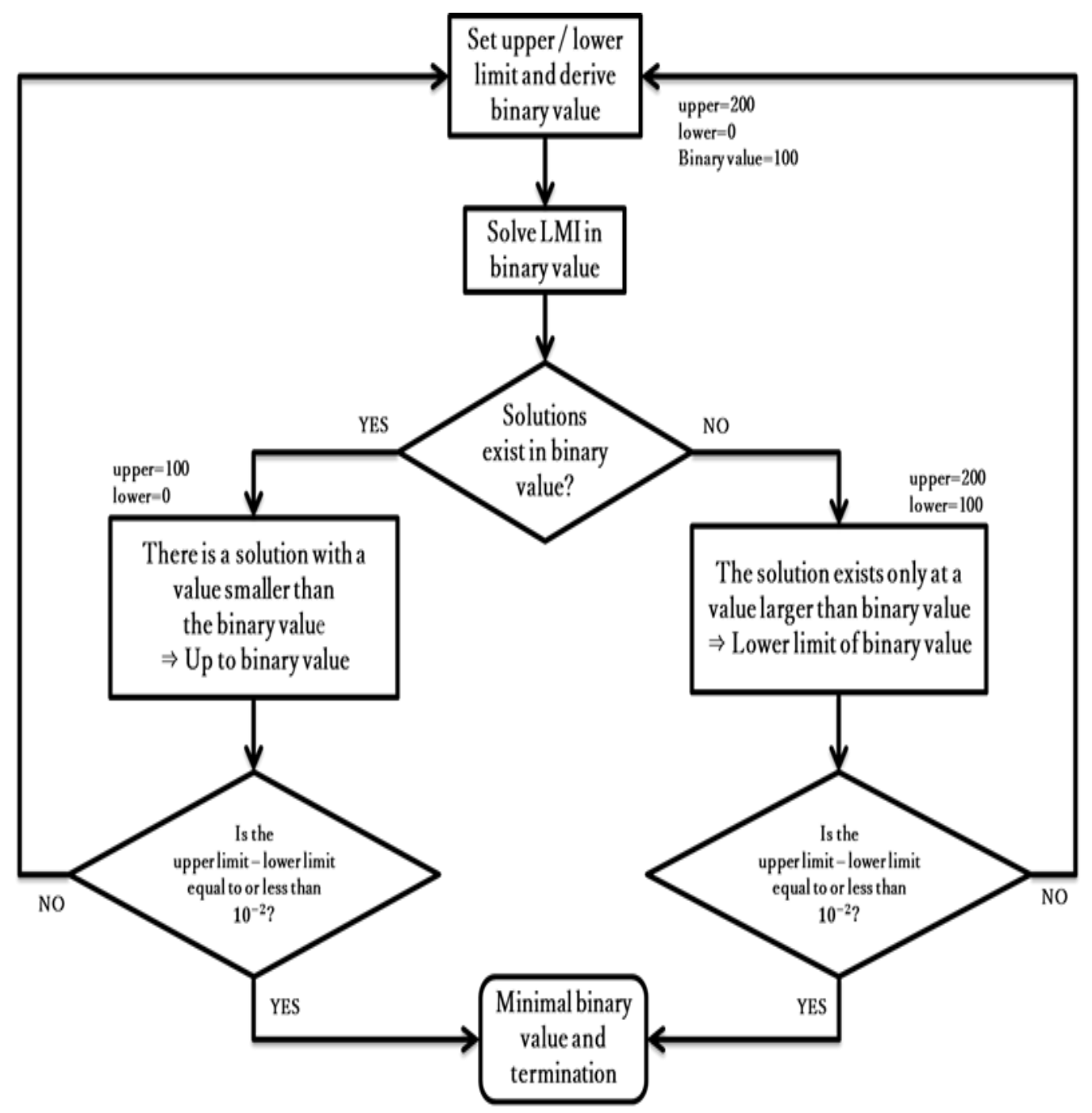

Figure 2 Binary search flow diagram. 


\subsection{Compensator design (approximate inverse system)}

In order to solve the tradeoff between attitude angle stability and velocity target value tracking, which is the purpose of this study, a feedforward pre-compensator was designed. By adding a compensator, the control amount is reduced by feedback and target value shaping, and a posture control system that simultaneously satisfies posture angle stability is guaranteed by a hybrid system with a compensator.

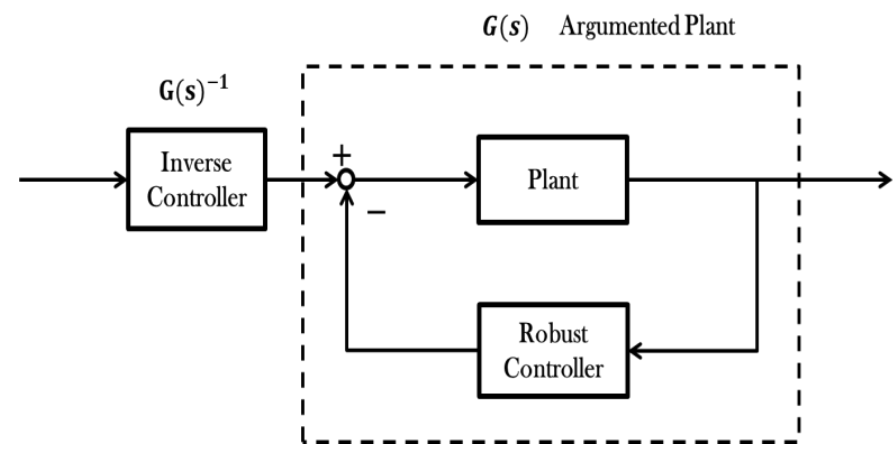

Fig.3 Hybrid system block diagram.

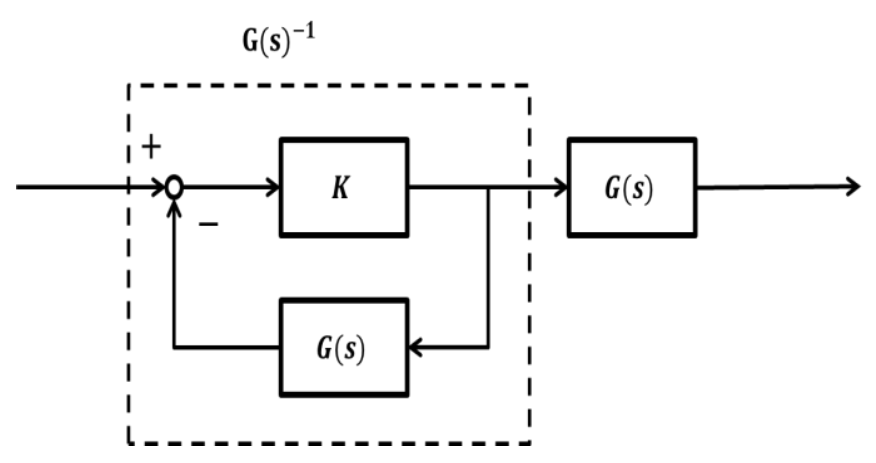

Fig.4 Inverse system block diagram.

In this study, the inverse system of the interference system is designed as a feedforward pre-compensator. There are a wide variety of design methods for inverse systems such as matrix manipulation, Massey-Sain algorithm, and neural networks (Sain \& Massey, 2008; Chunyan \& Aiguo, 2006). In this study, inverse systems are designed by high-gain approximation (Mezzi \& Soudani, 2016). The closed-loop system of the control plant and the 
feedback system is regarded as an augmented plant and an expanded plant, and the closed-loop system of the high gain and the expanded plant is designed as a pre-compensator for the interference system. By constructing a closed-loop system as shown in Fig. 3, it becomes possible to design an inverse system of an extended plant approximately, and an inverse model of the system can be derived (Buchholz \& Grünhagen, 2008; Murray-Smith, 2011). A controller that simultaneously satisfies the trade-off between the stability compensation and attitude stability improvement and wheel angular velocity target value shaping with respect to the road surface inclination angle disturbance by the hybrid system with the inverse system of the interference system and the robust control satisfying the $\mathrm{H}_{2}$ performance Design.

\section{Driving simulation}

In this study, a running simulation of a stable coaxial two-wheeled vehicle is realized by offsetting the target value to the rotational angular velocity of the drive wheel. In the first, 1 second, it is desired that the wheel accelerates to a rotational angular velocity of $300 \mathrm{deg} / \mathrm{sec}$, 6 seconds later from the start, it enters a slope with a road slope angle of 5 degrees. The simulation was designed to run or 11 seconds from the start, after which the wheel rotation angular velocity target value is reduced to $0 \mathrm{deg} / \mathrm{sec}$ to decelerate and stop.

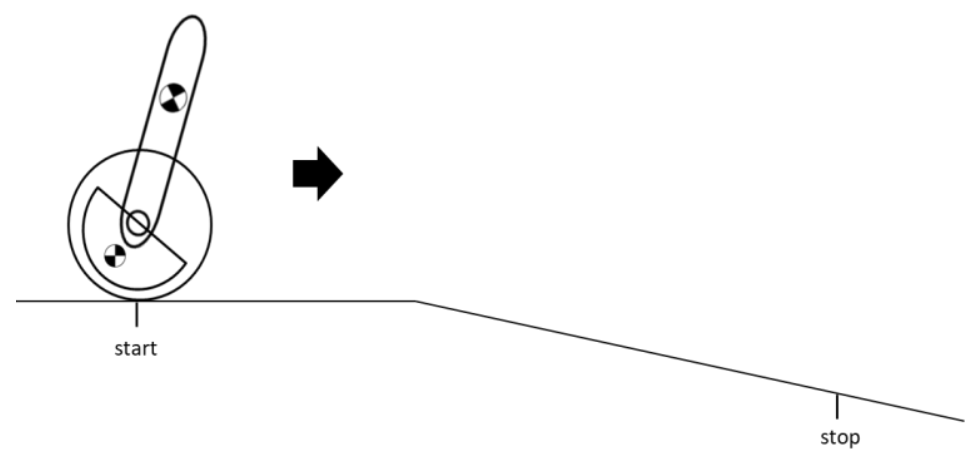

Figure 5 Slope simulation.

Figure 6 shows the tilt angle of the upper part of the car body, the tilt angle of the lower part of the car body, the angular velocity of the wheel, the input to the DC motor for the upper 
part of the car body, and the input to the DC motor for the wheel. The result of the slope running simulation by robust control is shown. Looking closely at the attitude angle of the upper part of the car body, it can be seen that it was possible to obtain a stability improvement in the attitude angle of the upper part of the car body using a robust controller as compared to the conventional method. However, looking at the wheel angular velocity target value, the tracking performance is deteriorated compared to the conventional method in terms of speed response and overshoot with respect to target value shaping. Therefore, a proposal to shape the target value with a compensator using an approximate inverse system was made.
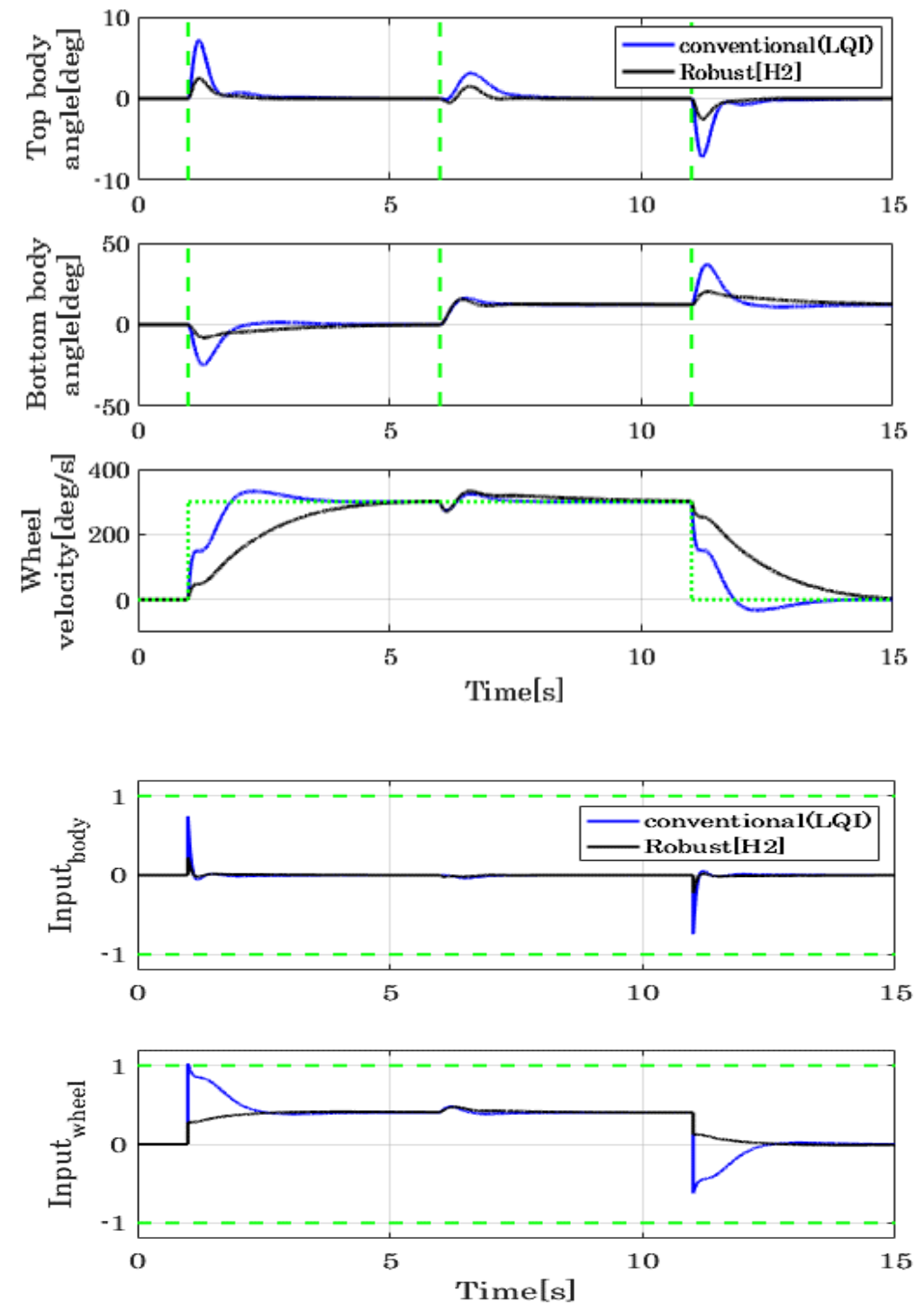
Fig.6 Simulation results (Robust control)
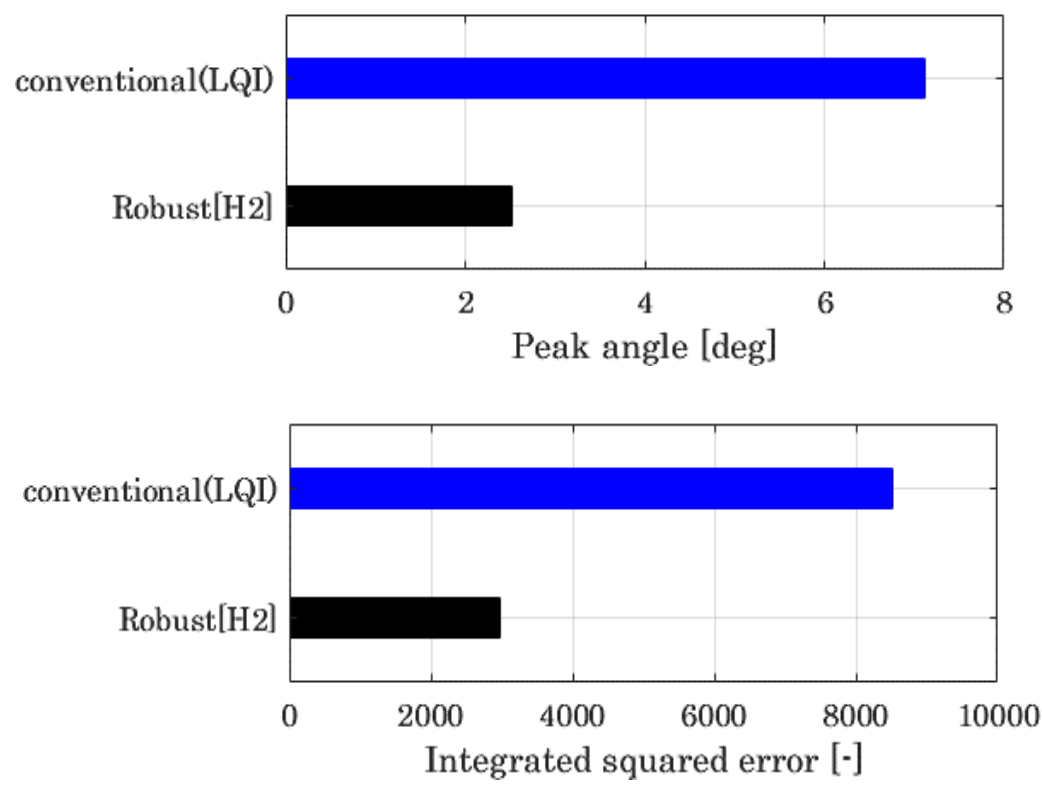

Figure 7: Peak/squared error (Hybrid system)

Figure 8 show the simulation results for the Hybrid system with the compensator by the inverse system with the robot following the path described earlier. It was confirmed that the target value can be shaped in the same way as the performance of the conventional method from the viewpoint of quick response and overshoot for the trade-off problem. Looking at the target value shaping and the attitude angle at the top of the vehicle body, improvement in attitude stability can also be observed. From the above, it can be confirmed that the trade-off problem between the posture angle of the vehicle body and the rotational angular velocity of the wheel has been solved. Therefore, in this study, a hybrid system with robust control with an inverse system as an attitude controller was considered for running on a slope in a bid to effectively improve the attitude stability and target value shaping. The input was also controlled without saturation, and the performance improvement was confirmed for the maximum posture angle and square area error, which are quantitative indicators of posture stability. 

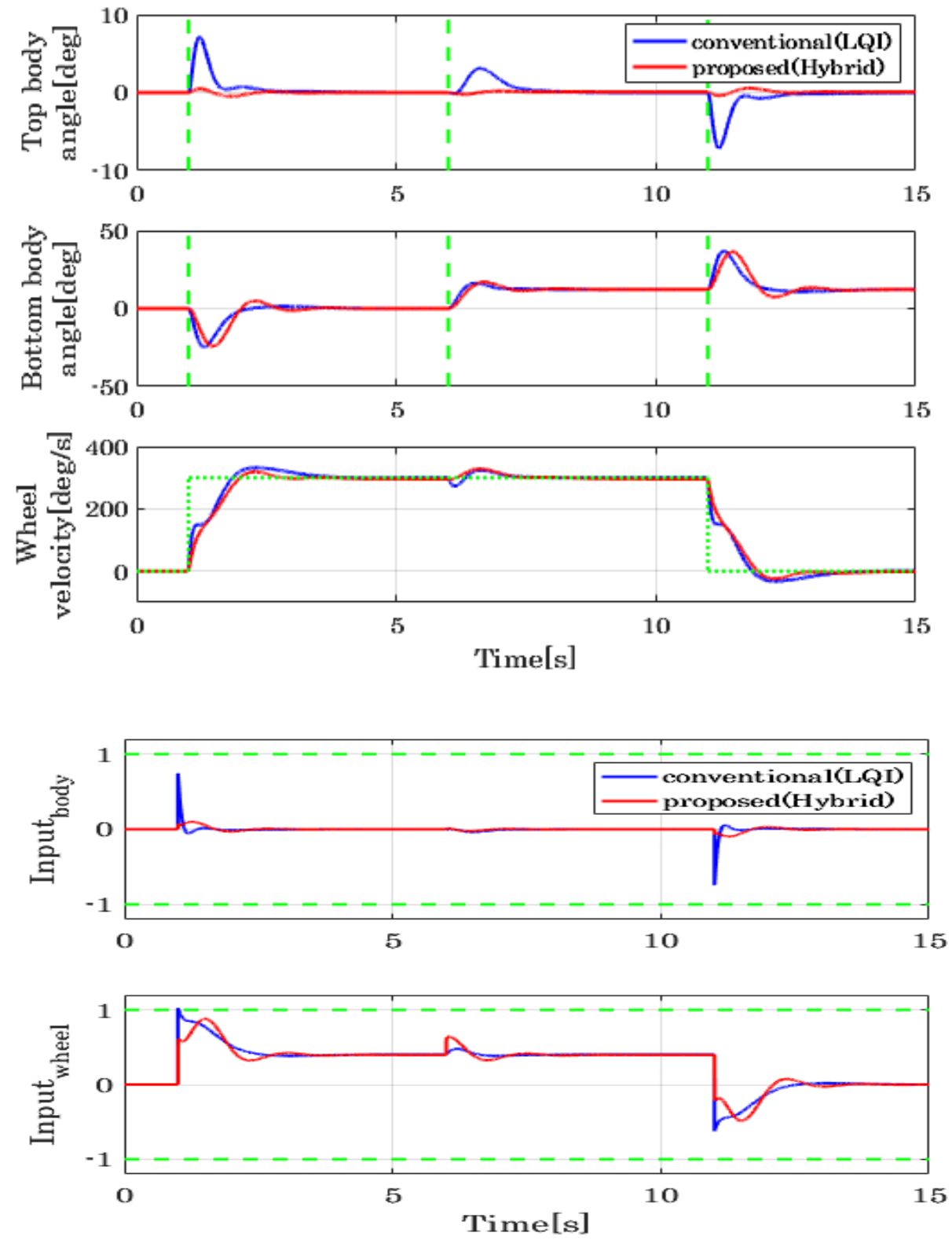

Figure .8 Simulation results (Hybrid system).

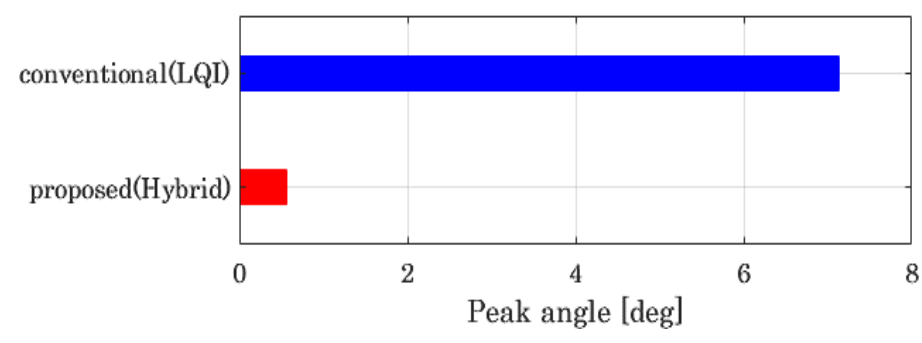




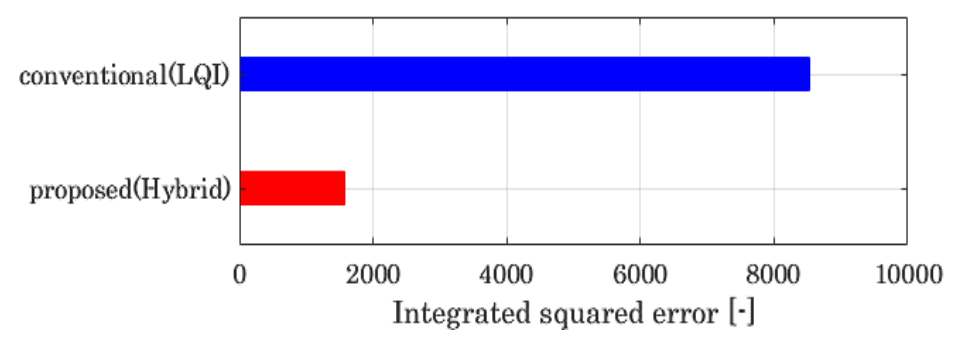

Figure 9: Peak/squared error (Hybrid system)

\section{Implementation environment construction}

Figure 11 shows the appearance and main dimensions of the stable coaxial two-wheeled vehicle used in this study. The center of gravity of the entire stable coaxial motorcycle is 64.7 $\mathrm{mm}$ below the center of rotation of the wheels. The drive mechanism at the top of the car body has a self-locking function by adopting a DC motor unit using a worm gear. The movable limit angle of the upper part of the car body is \pm 45 degrees in the front and back, and the total weight is $23 \mathrm{~kg}$. The upper part of the car body is a poster frame, which is a robot with an advertising function that can present information to the people around. This is because it is assumed to be used as a moving mechanism for service robots.

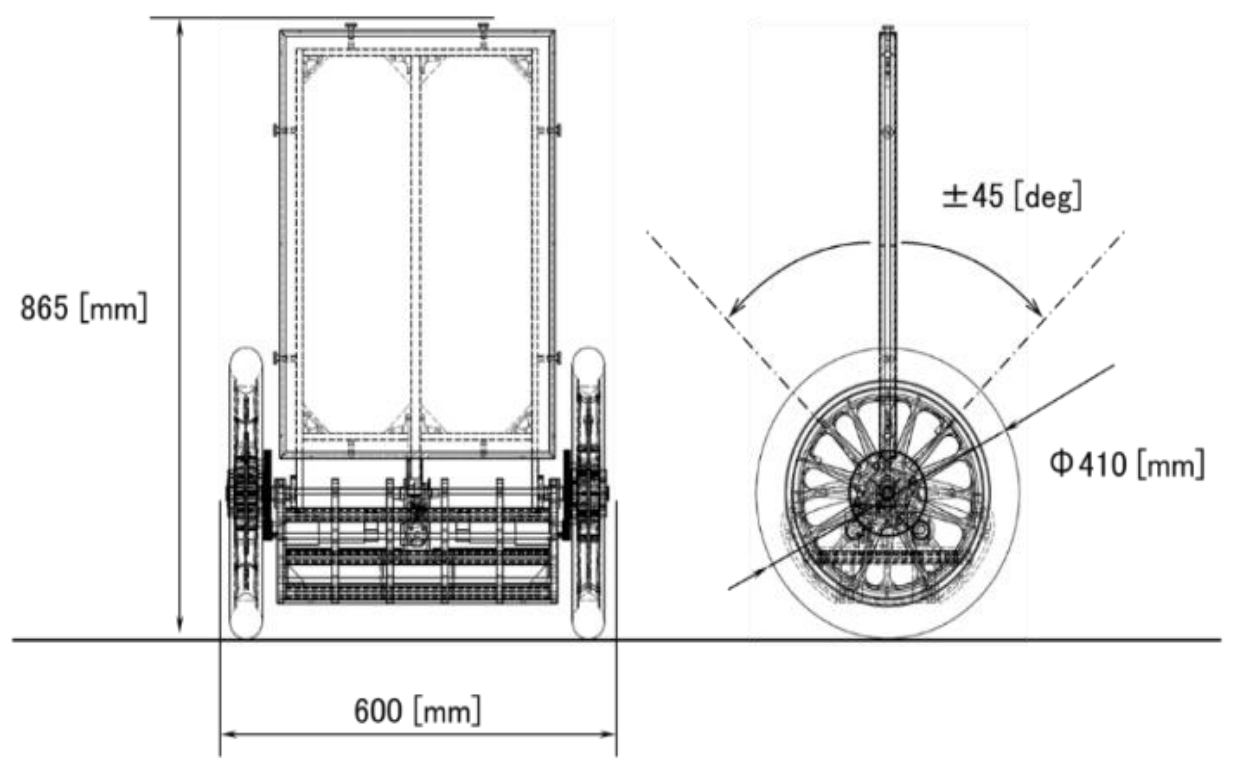

Figure 10 Prototype machine (front/side view) 


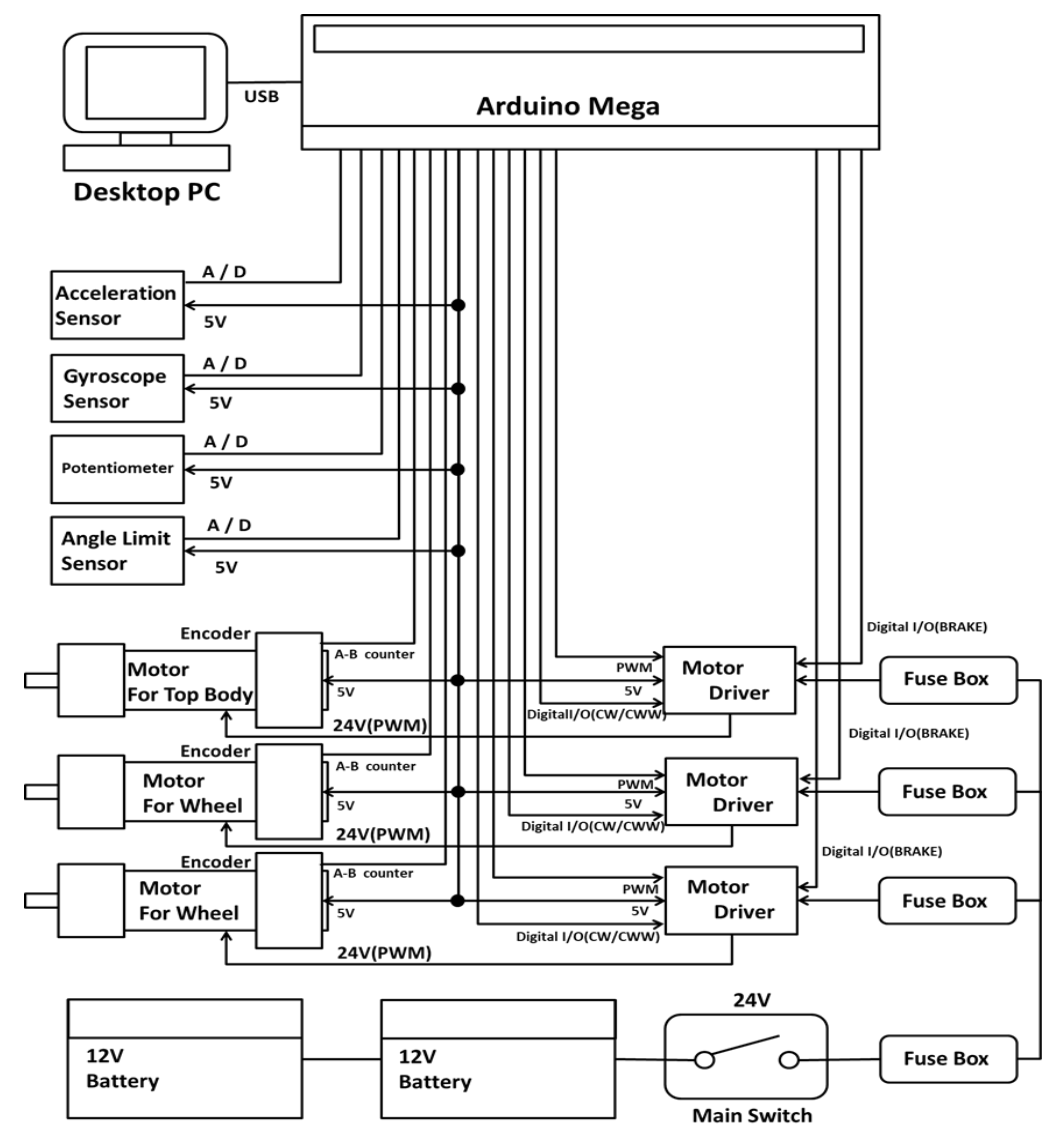

Figure 11 System configuration (Arduino).

This paper describes how to build an environment for model-based development with Arduino, which is the CPU used to build the implementation environment required for rapid prototyping (RCP) required for this experiment. In recent years, an open source model-based development (MBD) environment using Arduino microcomputers, which has been widely used as a microcomputer for electronic work, has been provided free of charge. The MBD development environment with Arduino is diverse, such as HILS (Hardware in the Loop System) environment by automatic code generation that generates Simulink model code from Matlab / Simulink and directly implements on Arduino, and RCP (Rapid Control Prototyping) by implementation model. The development environment can be constructed at a very low cost. In this study, an RCP environment that uses Arduino Mega as an input / output device from a host PC via a USB cable using Arduino Mega was implemented and compared the designed control systems. The system representation is as shown in Figure 12. 


\section{Driving experiment}

In order to confirm the performance confirmed by the simulation in the actual system, an implementation experiment was performed using a prototype. The experiment on the ramp was carried out using a veneer plywood experimental slope in the laboratory. The dimensions are $900 \mathrm{~mm}$ wide and $2700 \mathrm{~mm}$ long, including the ramp in the middle.

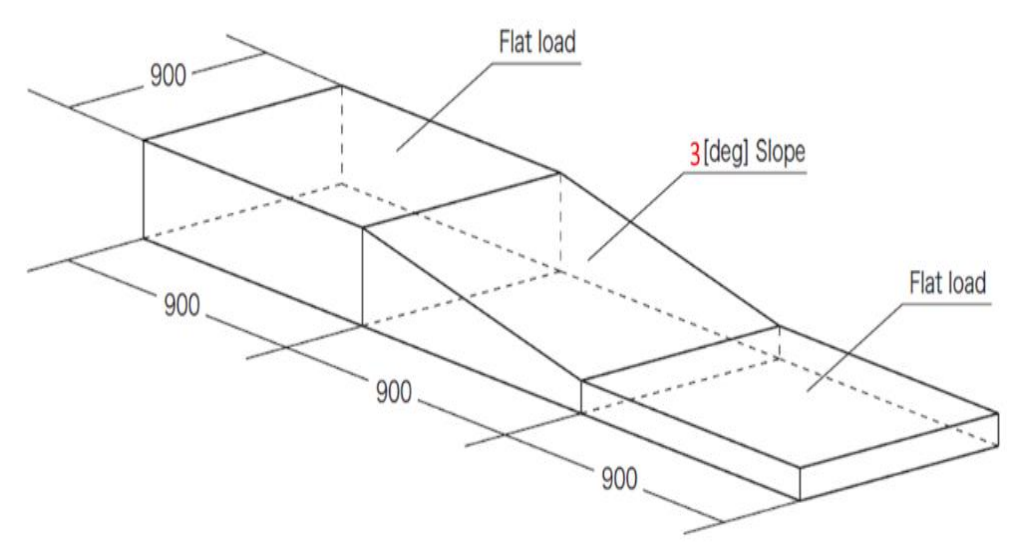

Figure 12 Slope for experiment.

Figure 14 shows the experimental results showing the posture angle of the top body, the posture of the bottom body, wheel velocity, input to the motor for the upper part of the car body, and the input to the DC motor for the wheel. From the figure, it can be seen that the conventional method has a large overshoot with respect to the target angular velocity of 50 [deg / s]. On the other hand, the Hybrid System, which is the proposed method, confirms that the vehicle is heading to the stop without overshooting after following the target value. As with flat roads, the stability of the posture angle is slightly improved compared to the conventional method, but with regard to target value shaping, it was confirmed that the follow-up performance with respect to target value shaping was improved. The control performance of the proposed hybrid type was confirmed through simulations and validated by experiments. The input is also were controlled without saturation. Although the maximum 
attitude angle and the square area error, which are quantitative indicators of attitude stability, are small, results with improved performance over the conventional method were obtained.
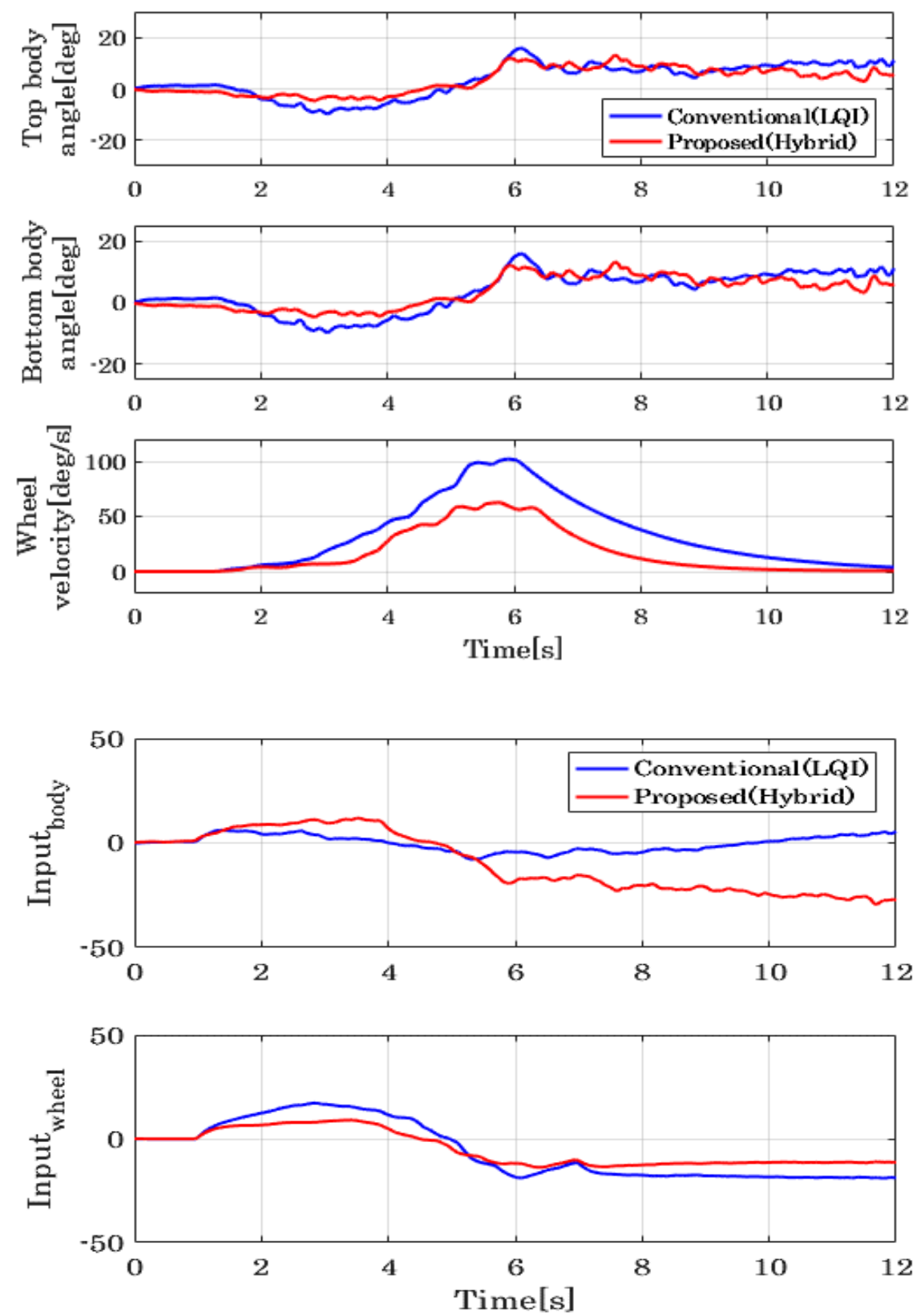

Figure 13 Experiment results (Hybrid system) . 

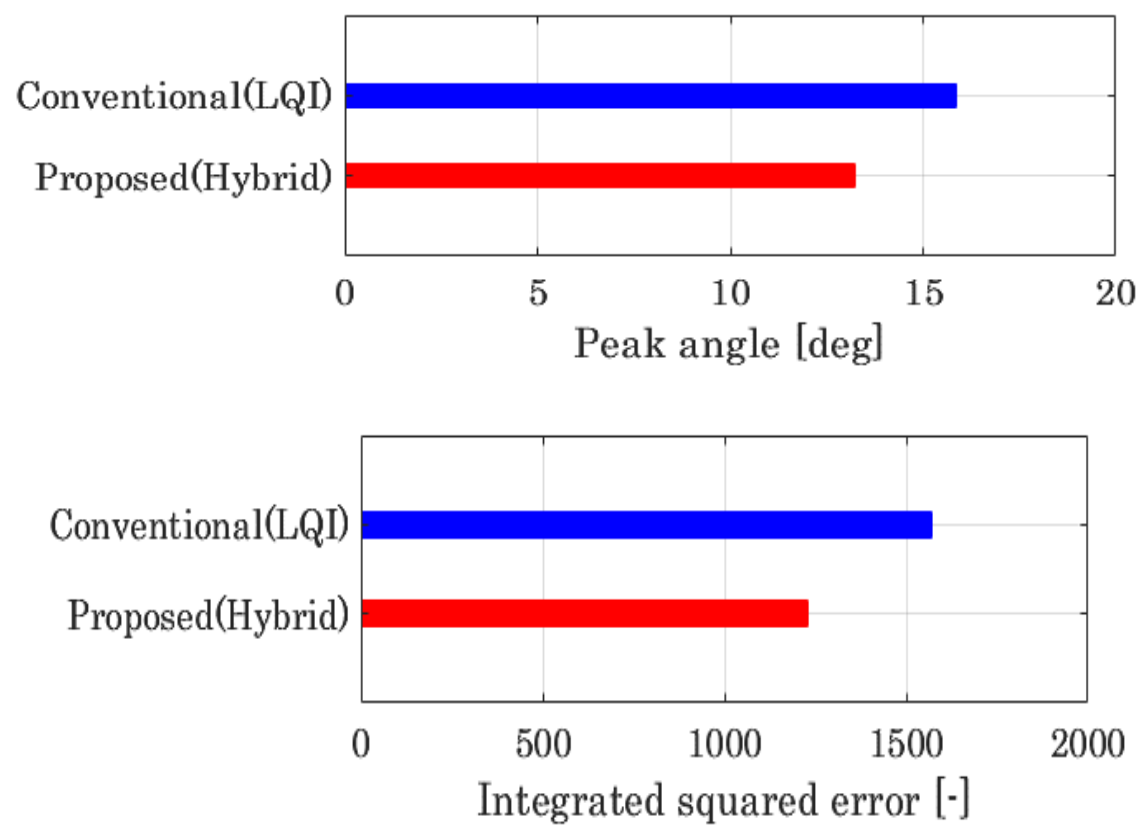

Figure 14 Peak/squared error (Hybrid system) .

\section{Conclusion}

In this paper, in order to improve stability in running on slopes, which has been a problem in previous research, and to solve the tradeoff between attitude angle stability and target value shaping, an interfering system reverse system and tilt angle disturbance compensation are provided. A controller with robust control was designed. In the simulation, the stability and follow-up results were improved from the viewpoint of attitude angle stability and speed target value shaping compared with the conventional method (LQI control). Also, in running tests with actual machines, both flat and inclined roads were successfully improved in stability compared to the conventional method. In terms of trackability to the speed target value, it was confirmed that the target value was tracked without overshooting when compared with the conventional method. Therefore, the proposed control method is very effective in eliminating the trade-off. The discussion on the actual machine is still shallow, and from the viewpoint of sampling time, there is a possibility that new problems and issues may arise depending on the control at higher speed processing. Therefore, deeper discussion 
on the actual machine such as the introduction of DSP for high-speed processing should be explored.

\section{Acknowledgement}

This work is partially supported by Grants-in-aid for Promotion of Regional Industry-University-Government Collaboration from Cabinet Office, Japan.

\section{References}

Buchholz, J. J., \& Grünhagen, W. v. (2008). Inversion Impossible?

Chan, R. P. M., Stol, K. A., \& Halkyard, C. R. (2013). Review of modelling and control of two-wheeled robots. In Annual Reviews in Control (Vol. 37, Issue 1, pp. 89-103). https://doi.org/10.1016/j.arcontrol.2013.03.004

Chunyan, D., \& Aiguo, W. (2006). Recurrent neural network-based inverse model learning control of manipulators. Proceedings of the World Congress on Intelligent Control and Automation (WCICA), 1, 2859-2863. https://doi.org/10.1109/WCICA.2006.1712887

Dai, F., Gao, X., Jiang, S., Guo, W., \& Liu, Y. (2015). A two-wheeled inverted pendulum robot with friction compensation. Mechatronics, 30, 116-125. https://doi.org/10.1016/j.mechatronics.2015.06.011

Deng, M., Inoue, A., Sekiguchi, K., \& Jiang, L. (2010). Two-wheeled mobile robot motion control in dynamic environments. Robotics and Computer-Integrated Manufacturing, 26(3), 268-272. https://doi.org/10.1016/j.rcim.2009.11.005

Jian-Wei, Z., \& Xiao-Gang, R. (2009). Flexible two-wheeled self-balancing mobile robot. IFAC Proceedings Volumes (IFAC-PapersOnline), 42(16), 117-124. https://doi.org/10.3182/20090909-4-JP-2010.00022

Liu, M., \& Dong, Z. (2018). Multiobjective robust H $2 / \mathrm{H} \infty$ fuzzy tracking control for thermal system of power plant. Journal of Process Control, 70, 47-64. https://doi.org/10.1016/j.jprocont.2018.08.004 
Mezzi, A., \& Soudani, D. (2016). Internal Model Control of A Class of Continuous Linear Underactuated Systems. INTERNATIONAL JOURNAL OF ADVANCED COMPUTER SCIENCE AND APPLICATIONS, 7(12), 140-148.

Murray-Smith, D. J. (2011). Feedback methods for inverse simulation of dynamic models for engineering systems applications. Mathematical and Computer Modelling of Dynamical Systems, 17(5), 515-541.

Sain, M., \& Massey, J. (2008). A modified inverse for linear dynamical systems. 51-51. https://doi.org/10.1109/sap.1969.269921 